\title{
Review of Suicide Prevention Programs: Massachusetts, United States, in Comparison with Seoul
}

\author{
Ji Hyun Baek ${ }^{1,2}$, Jong-lk Park ${ }^{3}$, Jeonghoon Ahn', Sung-won Roh', Jung-Yoon Heo', \\ Maurizio Fava ${ }^{6}$, David Mischoulon ${ }^{6}$, and Hong Jin Jeon ${ }^{1,6,7} \bowtie$ \\ 'Department of Psychiatry, Depression Center, Samsung Medical Center, Sungkyunkwan University School of Medicine, Seoul, Republic of Korea \\ 2Bipolar Clinic and Research Program, Massachusetts General Hospital, Harvard Medical School, Boston, MA, USA \\ ${ }^{3}$ Department of Psychiatry, Kangwon National University College of Medicine, Chuncheon, Republic of Korea \\ ${ }^{4}$ National Evidence-Based Healthcare Collaborating Agency (NECA), Seoul, Republic of Korea \\ ${ }^{5}$ Seoul National Hospital, Seoul, Republic of Korea \\ ${ }^{6}$ Depression Clinical and Research Program, Massachusetts General Hospital, Harvard Medical School, Boston, MA, USA \\ ${ }^{7}$ Departments of Medical Device Management and Research, Clinical Research Design and Evaluation, \\ Samsung Advanced Institute for Health Sciences \& Technology (SAIHST), Seoul, Republic of Korea
}

Suicide is a tragedy that has massive impact on society. In order to prevent suicide, active government intervention is necessary. The suicide rate in Seoul is rapidly increasing and is more than five times higher than that in the state of Massachusetts (MA) during the last decade, especially in the elderly. The suicide prevention program of MA is one of the most effective suicide prevention programs in the United States. The program views suicide as a preventable public health problem, and emphasizes treatment of depression and de-stigmatization of mental health illnesses to prevent suicide. Also, through active collaboration with mental health professionals, they try to identify atrisk populations and help them to get medical interventions. The program also actively collaborates with the regional coalition program and the Samaritans in taking care of the elderly, and supports the elderly in feeling worthwhile after retirement by helping them to work for communities as volunteers. For its part, the Seoul suicide prevention program puts more emphasis on "life respect culture" and "emotional support to high risk individuals by regular visiting". The annual budget of the Seoul suicide prevention program is one-quarter and that for mental health is about one-twentieth that of MA. Considering the high suicide rate and lower mental health service usage in Seoul, it is crucial to raise awareness of depression and decrease the stigma on mental illnesses. Furthermore, educational efforts with long-term investment in research on suicide are necessary.

Psychiatry Investig 2015;12(3):281-287

Key Words Suicide, Suicide prevention strategy.

\section{INTRODUCTION}

Suicide is emerging as a serious public health problem in Korea. Korea has the third highest suicide rate in both men and women and first in women globally. ${ }^{1}$ Completed suicide is only the tip of iceberg, and many more people think about and attempt to kill themselves. The lifetime prevalence of suicidal ideation, planning, and attempt in South Korea is reportedly $15.2 \%$, $3.3 \%$, and $3.2 \%$ (single $2.1 \%$ and multiple $1.1 \%$ ), respectively. ${ }^{2}$

Received: May 28, 2014 Revised: May 28, 2014

Accepted: August 27, 2014 Available online: July 6, 2015

$\triangle$ Correspondence: Hong Jin Jeon, $\mathrm{MD}, \mathrm{PhD}$

Department of Psychiatry, Depression Center, Samsung Medical Center, Sungkyunkwan University School of Medicine, 81 Irwon-ro, Gangnam-gu, Seoul 135710, Republic of Korea

Tel: +82-2-3410-3586, Fax: +82-2-3410-0050, E-mail: jeonhj@skku.edu

(c) This is an Open Access article distributed under the terms of the Creative Commons Attribution Non-Commercial License (http://creativecommons.org/licenses/by$\mathrm{nc} / 3.0$ ) which permits unrestricted non-commercial use, distribution, and reproduction in any medium, provided the original work is properly cited.
Suicide occurs due to complex interactions of various biopsycho-social problems. ${ }^{3}$ In order to prevent suicide, the systemic approach that requires active government intervention is necessary. The Commonwealth of Massachusetts (MA) is the third most densely populated US state and has about half the population of Seoul. The suicide rate in MA is one of the lowest in the US (47th out of 50 states) ${ }^{4}$ and about one-third of Seoul's (Table 1). The suicide prevention program of the MA state government is one of the most effective in the US. The MA suicide prevention program takes action through the MA Coalition for Suicide Prevention (MCSP), a community-based group that comprises suicide prevention advocates on the public and private sectors. The MCSP is actively working to prevent suicide through state-wide advocacy and collaboration.

In this paper, we will contrast the suicide prevention program of MA with Seoul's with the goal of improving the Seoul suicide prevention program. 
Table 1. Statistical values of Massachusetts versus Seoul

\begin{tabular}{|c|c|c|}
\hline & Massachusetts $s^{4,20}$ & Seoul $^{21}$ \\
\hline Area $\left(\mathrm{km}^{2}\right)$ & 27,336 & 605.21 \\
\hline Population (2012) & $6,646,144$ & $10,442,426$ \\
\hline Population density (persons $/ \mathrm{km}^{2}$ ) & 324 & 17,000 \\
\hline Median household income $(2012, \$)$ & 65,401 & 45,102 \\
\hline \multicolumn{3}{|l|}{ Annual budget } \\
\hline Total (2012, millions \$) & $30,597.92$ & $20,935.03$ \\
\hline Suicide prevention program $(2012$, million $\$)$ & $3.57^{*}$ & $0.95^{\ddagger}$ \\
\hline Mental health (2012, million \$) & $651.06^{\dagger}$ & $31.46 \S$ \\
\hline \multicolumn{3}{|l|}{ Suicide death (2011) } \\
\hline Total number & 553 & 2,722 \\
\hline Rates of both genders (100,000 persons) & 8.3 & 26.9 \\
\hline Rate of men (100,000 persons) & 14.7 & 37.4 \\
\hline Rates of women (100,000 persons) & 3.7 & 16.6 \\
\hline Gender ratio (male/female) & 4.0 & 2.3 \\
\hline The most common method & Hanging & Hanging \\
\hline Rank of causes of death & 12th & 4 th \\
\hline Ranking of suicide for each US state (2010) & $47 \mathrm{th} / 51$ & - \\
\hline
\end{tabular}

Suicide prevention program and mental health of Massachusetts has been supported by not only state budget but also many community volunteers and personal donations. *budget for Suicide Prevention and Intervention Program in Massachusetts, ${ }^{15}$ tbudget for Mental Health in Massachusetts, ${ }^{15} \neq$ budget for Suicide Prevention and Intervention Program in Seoul, ${ }^{16}$ \$budget for Mental Health in Seoul ${ }^{17}$

\section{HISTORY}

In 1958, the first suicide prevention center in the US opened in Los Angeles, California, with funding from the U.S. Public Health Service. In 1966, the Center for Studies of Suicide Prevention was established at the National Institute of Mental Health (NIMH) of the National Institutes of Health (NIH). ${ }^{5}$ Suicide was declared a major public health problem by the US Surgeon General, and federal/national expertise and resources have and continue to be invested to develop the national strategy for suicide prevention. ${ }^{5}$ The MA suicide prevention program, which takes charge of the state-level suicide prevention program, launched the MCSP in 1999. MCSP is a community-based group comprised of suicide prevention advocates including public and private agency representatives, non-profit organizations, policymakers, survivors of suicide loss, suicide attempt survivors, mental health clinicians and other health care providers, law enforcement, mental health and public health consumers, and concerned citizens. In 2002, MCSP released the first State Plan for Suicide Prevention and obtained firsttime legislative funds for suicide prevention. In September 2009, the Coalition launched the MA Strategic Plan for Suicide Prevention representing the diverse participation of over $500 \mathrm{Co}$ alition members. ${ }^{6}$

\section{STATISTICS OF SUICIDE}

Suicide is the fourth leading cause of death in the Korean population and the most common cause of death for people aged $10-40$ years. ${ }^{7}$ Although the suicide rates slightly decreased from 2011 to 2012 (from 31.7/100,000 to 28.1/100,000), ${ }^{7}$ Korea still has the highest suicide rate among the Organization for Economic Co-operation and Development (OECD) countries. In fact, Korea is the only country where the suicide rate has been increasing during last decade among the OECD countries. ${ }^{8}$

In 2010, 12.4/100,000 died by suicide in the US, ranking 10th of all causes of death..$^{9}$ The US suicide rate had been consistently decreasing until 2000 . Suicide rates in the US then rose mildly from $10.4 / 100,000$ in 2000 to $12.4 / 100,000$ in 2010, whereas the suicide rates in South Korea increased from 13.6/ 100,000 to $31.2 / 100,000$ in the same period. ${ }^{10}$

Although the suicide rates of MA and Seoul are both lower than the national suicide rates, the increase of the suicide rate in Seoul is more than five times higher than that of MA during the last decade (increase from 6.3/100,000 in 2000 to 9.1/ 100,000 in 2010 In MA; and from 9.7/100,000 to 24.3/100,000 in Seoul in the respective years) (Figure 1). In particular, the suicide risks in the elderly population are markedly higher in Seoul than in the MA (Figure 2). Notably, the suicide rate for women over 75 years of age was nearly $0 \%$ in MA. The suicide rate for females over 75 years of age in Seoul was three times 

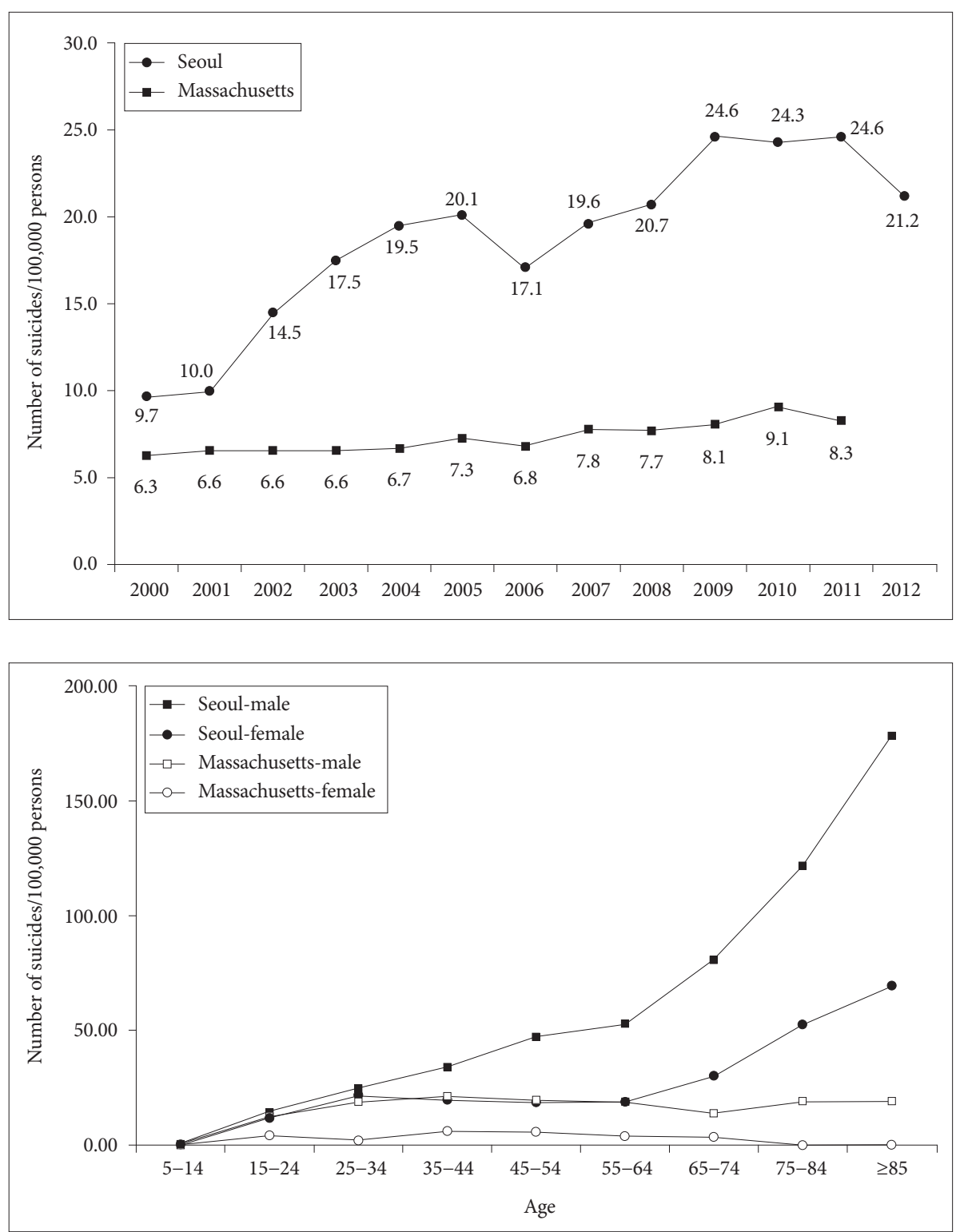

Figure 1. Annual suicide rates of Massachusetts versus Seoul. ${ }^{20}$ higher than for middle-aged females.

\section{OVERVIEW OF MISSION}

The MA suicide prevention program views suicide as a preventable public health issue and tries to reduce the number of suicides and suicide attempts among MA residents. They do this by 1) raising awareness of suicide as a public health issue; 2) providing support to communities, agencies and individuals interested in suicide prevention; 3) providing education and training for professionals and caregivers; 4) funding community-based suicide prevention and mental health promotion programs; 5) supporting and collaborating with state, regional and community suicide prevention coalitions; and 6) supporting and encouraging communities to collaborate across disciplines to prevent suicide and suicide attempts across the life span.

The Seoul suicide prevention programs focus on developing a suicide prevention strategy that is more applicable to the current situation in Korea. They seek to reduce the number of suicide victims by $50 \%$ by 2020 . They plan to do this by 1 ) establishing a "life respect culture" and improving awareness of mental health;2) improvement of mental health by age group and empowerment of response to stress; 3) facilitating community involvement in suicide prevention; 4) establishing a suicide prevention network in collaboration with private and public sectors; and 5) building scientific evidence for suicide prevention program. 


\section{DEPRESSION AND SUICIDE}

Mental disorders including depression, bipolar disorder, alcohol and substance use are well known risk factors for suicide. The MA government ${ }^{11}$ and the American Foundation for Suicide Prevention (AFSP), a non-profit organization to prevent suicide and help people who suffer from it, both try to increase awareness of the importance of mood disorder in suicide. The Suicide Prevention Resource Guide of the MA government reports that 1 ) the risk of suicide is increased by more than 50 percent in depressed individuals; 2) about 60 percent of suicides were in depressed people; 3 ) the risk of suicide in people with major depression is about 20 times that of the general population; 4) people with multiple episodes of depression are at a greater risk for suicide than those that have had one episode; and 5) a dependence on alcohol and drugs in addition to being depressed represents a greater risk for suicide. In particular, the guide states that people who are depressed and exhibit the following symptoms are at particular risk for suicide: 1) extreme hopelessness; 2) lack of interest in activities that were previously pleasurable; 3 ) heightened anxiety and/or panic attacks; 4) global insomnia; 5) talk about suicide or a prior history of

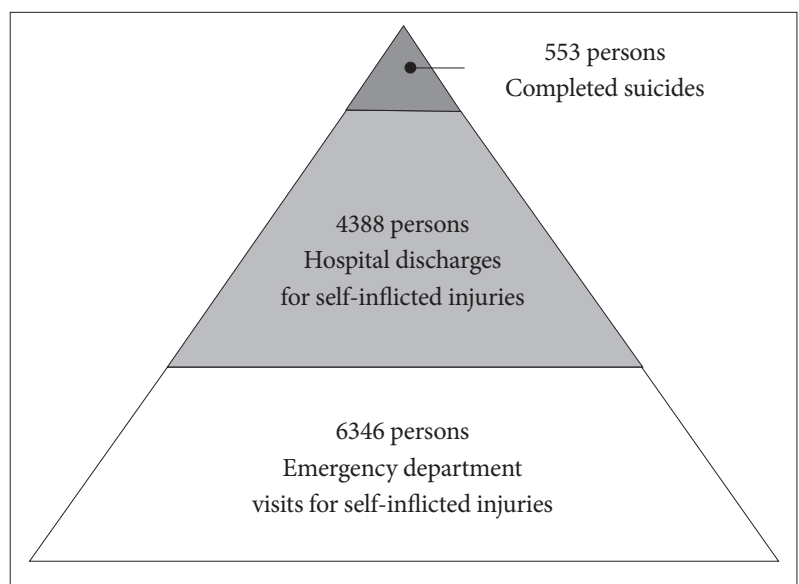

Figure 3. Number of completed suicides, hospital discharges, and emergency department discharges for nonfatal self-inflicted injury in Massachusetts, $2011{ }^{20}$ attempts/acts; and 6) irritability and agitation.

In MA, the number of people who visited the emergency room for treatment of self-inflicted injuries was 11 times higher than the number of completed suicides (Figure 3) and mental health problem and mental illness were the most common associated factor with suicide (Figure 4). Even under a highly stressful situation, most people who do not have a pre-existing mental disorder or other major risk factors do not show increased risk of suicide. Instead, exposure to extreme or prolonged environmental stress can lead to depression, anxiety, and other disorders that can, in turn, increase the risk for suicide. ${ }^{12}$

The Seoul suicide prevention programs also include screening and support for depression and mental health problem, but focus on mental health issues much less than in the MA program.

\section{DECREASING STIGMA ON MENTAL HEALTH ILLNESSES AND SUICIDE}

Although suicide occurs due to complex interplay of various causes, the single most important factor is undetected or untreated mental illnesses. Hence, decreasing the stigma concerning mental illnesses and raising awareness of the importance of the treatment are crucial in suicide prevention.

One of the main missions of the MA suicide prevention program is decreasing the stigma concerning mental health illnesses and suicide. In collaboration with the MCSP, they have sponsored annual Suicide Prevention Awareness Events. Also, through active collaboration with mental health professionals, they try to screen at-risk populations and help them to get medical help.

The Seoul suicide prevention program also tries to increase the awareness of suicide; however, it puts more emphasis on "life respect culture", i.e., a culture in which respect for life is considered important. A previous Korean national study showed that individuals with depression had more permissive attitude to suicide, i.e. regarding suicide as free from life suffering, a personal right, and a solution to a difficult situation, ${ }^{13}$ compared to

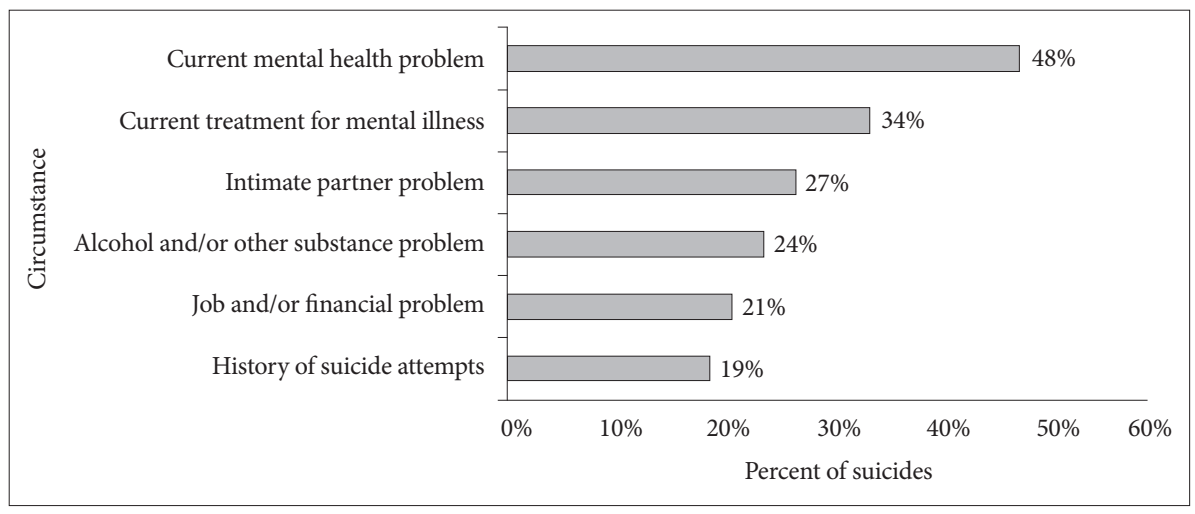

Figure 4. Circumstances associated with suicide in Massachusetts, $2010 .^{20}$ 
those without depression. However, that does not mean that suicide attempters do not respect life. According to the recent report by the Korean Ministry and Welfare, depression and psychiatric symptoms are the most common cause of suicide attempts, and suicide attempters have 25 times higher risks for completed suicide than general population in Korea, ${ }^{14}$ which corroborates with findings from MA. However, Koreans still use mental health services less than Americans. Koreans have a very strong stigma concerning mental health illnesses and use of mental health services, which can be a great barrier to seek help. Raising awareness of mental illness and decreasing barriers to use of mental health services are important in suicide prevention.

\section{COALITION PROGRAM}

The MA suicide prevention program puts much emphasis on the coalition program to bring together people from different fields and backgrounds to reduce suicide and promote mental health. They actively take part in the state-wide suicide prevention program. The MCSP leads the developing, updating and expanding, monitoring and evaluating of the strategic plan of the MA suicide prevention program. It also develops the State Plan to share with the state legislature, appropriate state agencies and other stakeholders in collaboration with the MA suicide prevention program.

Given the importance of collaboration between private and public sectors, the Seoul suicide prevention program also tries to establish such a coalition. In order to engage local communities in the program, suicide survivor advocacy groups were recruited since 2011. So far, however, the Seoul municipal government has taken the lead in most aspects of the suicide prevention program from initial planning to evaluation. Since the Seoul suicide prevention program is still in its early stages of development, it might be too early to involve the private sectors actively in the program. But as the program matures, active involvement of the private sector will be key to success.

\section{BUDGET}

The annual budget of the MA suicide prevention program was 3,569,444 U.S. dollars in 2012, which accounted for $0.01 \%$ of the total annual budget of the MA state government. ${ }^{15}$ About $4 \%$ of the budget was used for a grant to support the community-based suicide prevention and mental health promotion program. The suicide prevention program of MA has been supported by not only the state budget but also by many community volunteers and personal donations. The annual budget of the Seoul suicide prevention program was 1,026,000,000 won (about 954,418 USD) in 2012, which accounted for $0.005 \%$ of the total annual budget of Seoul (Table 1). ${ }^{16}$

The budget for mental health shows obvious differences between MA and Seoul. The annual budget of MA for mental health was 651.06 million USD in 2012, and half of the budget, 329.29 million USD, was allocated for the Mental Health Services including adult, homeless and emergency support. ${ }^{15}$ On the other hand, the annual budget of Seoul for mental health services was 32,735,000,000 won (about 31.46 million USD). ${ }^{17}$ The annual budget of the Seoul suicide prevention program is thus only $1 / 4$ (nominal) of MA's budget and that of mental health is $1 / 21$ (nominal) of MA's. Even after adjusting for purchasing power, the Seoul suicide program annual budget is 36\% that of the MA program and the one for mental health is $6.3 \%$ that of the MA.

\section{INVESTMENT IN RESEARCH}

To prevent suicide, it is important to know why people commit suicide. The MA suicide prevention program seeks to plan evidence-based strategy and education. Both the MA and Seoul prevention programs regard research on suicide as an important part in suicide prevention.

To promote scientific research on suicide, various funding source is available in the US. The National Institutes for Mental Health (250,000 dollars per project), the Centers for Disease Control and Prevention (4.1 million dollars over 5 years per project) and the US Army (about 10 million dollars per project) have separate grants for research on suicide.

In addition to that, the AFSP, a US non-profit organization, works to understand and prevent suicide, and to help those who suffer from suicide. ${ }^{12}$ The AFSP funds six categories of research every year from 30,000 to 225,000 dollars per project.

In Korea, there is no available research grant specifically targeted to studies on suicide. In 2013, Korea's research and development $(\mathrm{R} \& \mathrm{D})$ budget for mental health comprised $2.4 \%$ of the total public health and medical R\&D budget $(309400$ million won). The R\&D budget for mental health decreased relatively from previous years when compared to the overall increase of the total public health and medical R\&D budgets.

In order to prevent suicide, it is important to invest a sufficient amount of the budget continuously in suicide research. However, regular funding for suicide research is still insufficient in Korea.

\section{EDUCATION}

The MA suicide prevention program supports education and training in suicide prevention. In collaboration with the MCSP, it tries not only to educate local communities but also to train religious and social organizations. In addition, it also provides education for mental health professionals who work 
directly in the assessment and management of suicidal patients.

The Seoul suicide prevention program also has plans to train mental health professionals, elderly caregivers (both from medical and social services) and any volunteers to work with at-risk populations as gatekeepers to prevent suicide. But it still lacks a long-term plan for training volunteers and running the overall education process.

\section{ELDERLY SUICIDE}

The elderly are considered especially at risk of suicide. Although older adults attempt suicide less often, they tend to complete suicide more often than other age groups. However, the elderly suicide rate in MA has remained lower than other age groups. The MA suicide prevention program actively collaborates with the regional coalition program and the Samaritans in taking care of the elderly. The Samaritans is a non-profit organization to reduce the incidence of suicide by alleviating suicidal ideation and helplessness among individuals in the community. ${ }^{18}$ Their programs for the elderly are funded by the MA suicide prevention program, and provide direct suicide prevention and wellness workshops to seniors in independent living centers throughout the Greater Boston area. Also, it provides befriending call services from trained volunteers who are also seniors and they support the elderly to be free of a feeling of worthlessness after retirement by way of helping them to work for communities as volunteers.

The Seoul suicide prevention program plans preventive approaches that integrate public health and socialcare. It tries to develop the suicide surveillance system to utilize mental health professionals, medical and social caregivers. Also, it also plans befriending phone support and caregiver services for elders who live alone. However, providing emotional supports by regular visits are the initial plans for the elderly. Other than that, detailed care plans are lacking in the Seoul suicide prevention program.

\section{VETERANS}

Veterans are important at-risk population for suicide. The MA suicide prevention programs provide a Crisis Hotline for Veterans. Counselors and outreach programs contracted by the department staff the crisis hotline. When veterans who seek counseling programs or concerned family members of those veterans contact the hotline, they are directed towards the programs and services offered by their local or regional veteran office. Workers at the hotlines are trained in issues of mental health counseling and veterans services. ${ }^{19}$ The Seoul suicide prevention program does not cover programs for veterans.

\section{CONCLUSION}

Suicide is a tragedy that has massive impact across the whole society. In order to reduce the suicide rate, it is important to realize that suicide is a preventable public health issue and to develop preventive strategies in collaboration with various stakeholders including public health administrators, mental health professionals and concerned citizens. In MA, the suicide prevention program tries to reduce the incidence of suicide by the coalition program, public education and investment in research. So far, it has shown to be effective in reducing the incidence of suicide. The Seoul suicide prevention program also puts much effort into reducing suicide, but is still at its beginning and has room for improvement. Considering the high suicide rate and lower mental health service usage, it is crucial to raise awareness of depression and decrease the stigma of mental illness in Korea. Also, more active participation of the local community in planning, conducting and evaluating prevention strategies is necessary. In addition, educational efforts with long-term investment in research on suicide are still somewhat lacking. Although people are becoming more aware about suicide in Korea recently, suicide is still classified as "intentional self-harm" by national statistics. Suicide is not an intentional act, but the result of untreated or difficult-to-treat psychiatric disorders including depression and substance use. Long-term investment in building infrastructure, professional training and research are warranted to reduce the number of people who suffer as a result of suicide.

\section{Acknowledgments}

This research was supported by the Small Research Society Fund from the Policy Research Institute of the Korean NeuroPsychiatric Association. It was also supported by the Basic Science Research Program through the National Research Foundation of Korea (NRF) funded by the Ministry of Education, Science and Technology (No. 2011-0013064) and the Samsung Medical Center Clinical Research Development Program (CRDP) Grant SMO1131461. We thank Mr. Alan Holmlund, Director, and Ms. Christine M. Farrell-O'Reilly, Deputy Director of Suicide Prevention Program, Massachusetts Department of Public Health for their great comments and review the manuscript.

\section{REFERENCES}

1. WHO Worldwide Initiative for the Prevention of Suicide. WHO Suicide Prevention. Geneva: World Health Organization; 2011.

2. Jeon HJ, Lee JY, Lee YM, Hong JP, Won SH, Cho SJ, et al. Lifetime prevalence and correlates of suicidal ideation, plan, and single and multiple attempts in a Korean nationwide study. J Nerv Ment Dis 2010;198:643646.

3. Botsis AJ, Soldatos CR, Stefanis CN. Suicide: Biopsychosocial Approaches. Amsterdam: Elsevier; 1997.

4. McIntosh JL, Drapeau CW. U.S.A. suicide 2010: Official Final Data. Washington, DC: American Association of Suicidology; 2012.

5. Office of the Surgeon General (US) and National Action Alliance for Suicide Prevention (US). 2012 National Strategy for Suicide Prevention: Goals and Objectives for Action: A Report of the U.S. Surgeon General and of the National Action Alliance for Suicide Prevention. Appendix 
C Brief History of Suicide Prevention in the United States. Washington, DC: US Department of Health \& Human Services; 2012.

6. The Massachusetts Coalition for Suicide Prevention. About the MCSP. http://www.masspreventssuicide.org/about-the-mcsp. Boston: The Massachusetts Coalition for Suicide Prevention; 2014.

7. Statistics Korea. 2012 Death and Cause of Death in Korea. Daejeon: Korea National Statistical Office; 2013.

8. Organisation for Economic Co-operation and Development. OECD Health Data 2008: Statistics and Indicators for 30 Countries. Washington, DC: OECD Publishing; 2008.

9. Murphy SL, Xu JQ, Kochanek KD. National Vital Statistics Reports. Deaths: Final Data for 2010. Hyattsville: National Center for Health Statistics; 2013.

10. Statistics Korea. 2010 Death and Cause of Death in Korea. Daejeon: Korea National Statistical Office; 2011.

11. Suicide Prevention Program. Massachusetts Suicide Prevention Resource Guide. Boston: Massachusetts Department of Public Health; 2008.

12. Prevention AFS. Risk Factors and Warning Signs for Suicide. New York: American Foundation for Suicide Prevention; 2014.

13. Jeon HJ, Park JH, Shim EJ. Permissive attitude toward suicide and future intent in individuals with and without depression: results from a nationwide survey in Korea. J Nerv Ment Dis 2013;201:286-291.
14. Korean Ministry and Welfare SNU. National Suicide Survey. Daejeon: Korean Ministry and Welfare; 2014.

15. The Commonwealth of Massachusetts. FY2012 Budget Summary of Massachusetts (http://www.mass.gov/bb/gaa/fy2012/). Boston: The Commonwealth of Massachusetts; 2014.

16. Seoul Metropolitan Government. 2012 Budget for Suicide Prevention in Seoul (http://health.seoul.go.kr/archives/3756). Seoul: Seoul Metropolitan Government; 2014.

17. Seoul Mental Health Statistics. 2012 Budget for Mental Health Services of Seoul (http://www.seoulmentalhealth.kr/reference/subject.jsp?open Mode=qut1_1\#). Seoul: Seoul Mental Health Improvement Center; 2014.

18. Massachusetts Suicide Prevention Resource Guide. Boston: Suicide Prevention Program Injury Prevention and Control Program Massachusetts Department of Public Health; 2008.

19. Veterans Crisis Line. Washington DC: U.S. Department of Veterans Affairs; 2014

20. Injury Surveillance Program. Suicides and Self-Inflicted Injuries in Massachusetts: Data Summary. Boston, MA: Department of Public Health; 2013.

21. Statistics Korea. 2011 Death and Cause of Death in Korea. Daejeon: Korea National Statistical Office; 2012. 